\title{
Optimizing the Radiology Experience through Radiologist-Patient Interaction
}

Andrew W. Phillips ${ }^{1}$, Rebecca A. Landon ${ }^{1}$, Gregory S. Stacy ${ }^{2}$, Larry Dixon ${ }^{2}$, Andrea L. Magee ${ }^{2}$, Stephen D. Thomas ${ }^{2}$, Xi Dai ${ }^{2}$, Christopher Straus ${ }^{3,2}$

1. Emergency Medicine, University of North Carolina, Chapel Hill, USA 2. Radiology, The University of Chicago Medicine, Chicago, USA 3. Nuclear Medicine, Thoracic Imaging, The University of Chicago Medicine, Chicago, USA

Corresponding author: Christopher Straus, cstraus@uchicago.edu

\section{Abstract \\ Objective}

The goal of this survey-based study is to explore patients' knowledge of and expectations for radiologists in the outpatient setting.

\section{Materials and Methods}

A comprehensive survey was distributed to adult patients undergoing knee magnetic resonance imaging (MRI) over a one-year period from September 2015 through August 2016 at an urban, quaternary care academic medical center.

\section{Results}

The survey results demonstrate that only a subset of patients undergoing knee MRI at the institution during the survey period are aware of the role of the radiologist, which is a well-documented fact described in the literature. Approximately one-third of patients expected to meet the radiologist during their visit to the department of radiology to undergo a knee MRI. The vast majority of patients surveyed wanted to be able to contact the person who read their exam, but only one patient actually contacted the radiologist during the study period.

\section{Conclusion}

While the vast majority of surveyed patients wanted to be able to contact the person who read their knee MRI, only one patient actually did reach out to the radiologist to discuss findings. However, six of 36 followup respondents reported that they had contacted the person "who interpreted/read your exam:" two in person, one by email, three by phone, and one by other. Survey results demonstrated that only a subset of patients correctly understood the role of the radiologist ( $46 \%$ in the $1^{\text {st }}$ survey and $63 \%$ in the $2^{\text {nd }}$ survey, which does not represent a statistically significant difference), which suggests that perhaps the patients did have a conversation with a member of the radiology department staff whom they believed was actually the radiologist. The fact that patients expressed a desire to communicate with the person reading their reports, but then did not take advantage of the opportunity to contact the radiologist, suggests that the issue is more complicated than just a lack of a pathway for communication between patients and radiologists. Perhaps the lack of a clear understanding of the role of the radiologist hinders patients from contacting radiologists, as they feel uncertain as to whom they are actually attempting to reach. Or perhaps patients are sufficiently reassured by having a means through which they could contact the radiologist and do not require the actual communication in order to feel comfortable. There remains a significant amount of work to be done in understanding the barriers in patient-radiologist communications.

Categories: Medical Education, Radiology

Keywords: radiology, patient education, mri knee

\section{Introduction}

Reporting practices in radiology represent a critical component of the patient's medical record in both the inpatient and outpatient settings. There has been considerable interest within the radiology literature to evaluate the efficiency of radiology reporting from the standpoint of the referring physician, patient, and radiologist [1-4]. Specific attention has been given to the matter of how and by whom results are communicated to the patient, with the recent emphasis on autonomy and patient centeredness.

Despite the advent of electronic medical records and implementation of patient portals, patients remain generally dissatisfied with how they receive their medical imaging results [1-4]. Patients want access to relatively detailed information with minimal delay [5-7]. Recent literature demonstrates that patients prefer to receive their imaging results directly from the referring physician and would like both the report and the 
Public awareness of the radiologist's role in their care, however, is limited, with only a subset of patients correctly identifying the role of the radiologist in several recent surveys addressing this issue [7,10]. The lack of awareness of the radiologist's role in these studies is understandable considering that radiologists often operate outside of direct patient interaction. Some studies, however, have shown that patients felt positive toward meeting the radiologist interpreting their examination, and this is the patient attitude that this study sought to explore [10]. Thus, this study sought to evaluate a simple communication intervention to improve patients' understanding of the radiologist's role and their satisfaction with the medical imaging communication process.

\section{Materials And Methods Study population}

This study was approved by the University of Chicago's institutional review board (IRB) as an IRB exemption and was HIPAA compliant. All adult patients over the age of 18 undergoing knee MRI during the data collection period from September 1, 2015 through August 16, 2016 were eligible. Patients without a known email address were excluded since this was a primary means of communication. Please see Appendix A for a sample communication.

\section{Survey design}

The survey was designed and implemented by the radiology department staff and a statistician. Four basic areas were assessed through the survey: 1) patient demographic information, 2) patient knowledge of radiology including perception of the radiologist's role, 3) patient opinions on methods for communication of test results, and 4) patients' perceived ease of access to their own health care record. Please see Appendix B for the complete survey instrument.

\section{Survey protocol}

Patients were contacted to obtain consent at least one business day prior to their scheduled MRI appointment. Following consent, a pre-appointment survey was administered by email. The preappointment survey restated that the subject's participation was voluntary, could be terminated at any time, and would have no effect on clinical care. To incentivize participation, entry into a drawing for a small prize was offered to patients upon completion of the survey.

The participating patients underwent the scheduled knee MRI as per the standard hospital radiology department protocol. MRI knee protocol comprises a group of MRI sequences to routinely assess the internal pathology of the knee. Within two to four business days following the MRI, participating patients received a copy of the diagnostic report with the intervention: a cover letter introducing the attending radiologist who interpreted the MRI (including a photograph, greeting from the radiologist and invitation to communicate directly with the radiologist about the interpretation). Please see Figure 1 of Appendices for the complete cover letter. In this written communication, patients were encouraged to contact the radiologist with any questions or comments regarding the imaging study or the overall experience in the radiology department. A second post-appointment survey was administered to patients two weeks after the date of the MRI. Collected survey data and exchanges with the radiologists were processed through a departmental data broker (Human Imaging Research Office (HIRO)) for the diagnostic reports.

\section{Statistical analysis}

Data were collected via REDCap (REDCap Consortium, Nashville, TN) and analyzed in SPSS v24 (IBM Corp, Armonk, NY). Descriptive statistics and univariate analyses were used as indicated. Chi-square was used for nominal comparisons and independent t-tests for continuous variable comparison. Nonresponse bias for the surveys was evaluated with a population comparison against age and sex of all knee MRIs performed during the study period [11].

\section{Results}

One hundred thirty-five patients were contacted, of whom, 63 declined to participate, three were excluded for not having an email address, and 69 consented to participate. Forty-nine consented participants responded at least partially to the first survey, and 36 consented participants responded at least partially to the follow-up survey, yielding a response rate of 25.6\% (AAPOR response rate definition \#4). Four musculoskeletal fellowship-trained attending radiologists participated. Three of the 135 potential respondents who were contacted did not have an email address and were excluded. Using the same percentage, we estimated that $2.3 \%$ of potential respondents who did not respond would also be ineligible. This is accounted for in the response rate calculation per the AAPOR guidelines [11].

\section{Demographics}




\section{Cureus}

The majority of the 49 respondents were female (71.4\%), and the mean (standard deviation) age was 42.4 (14.6). Most (95.9\%) of the 49 respondents had prior medical imaging, and $71.4 \%$ had a prior MRI. Almost a third of the total (49) respondents reported highest education obtained to be a graduate degree (30.6\%), $20.4 \%$ a bachelor's degree, $40.8 \%$ an associate's degree or "some college," and $8.2 \%$ a high school degree or equivalent.

\begin{tabular}{|l|l|}
\hline Characteristic & $\mathbf{n}(\%)$ or $\mathbf{n} \pm \mathbf{S D}$ \\
\hline Age & $42.4 \pm 14.6$ \\
\hline Hemale & $35(71.4)$ \\
Had prior MRI & $47(95.9)$ \\
\hline Highest education obtained & $35(71.4)$ \\
\hline High school or GED & \\
\hline Some college & $4(8.2)$ \\
\hline Associate's degree & $17(34.7)$ \\
Bachelor's degree & $3(6.1)$ \\
Graduate degree & $10(20.4)$ \\
\hline
\end{tabular}

\section{TABLE 1: Respondent demographics}

$\mathrm{SD}$, standard deviation; GED, graduate equivalent degree; MRI, magnetic resonance imaging

\section{Role of the radiologist}

Fewer than half (47.9\%) of 48 respondents initially reported that a radiologist is a "medical doctor." After receiving the cover letter with information about the radiologist and an invitation to communicate with him or her (the intervention), $69.4 \%$ of 36 respondents reported that they thought a radiologist was a "medical doctor," which was not significantly different from before the intervention, regardless of whether or not “don't know" responses were included $\left(X^{2}(2)=4.233, p=0.120\right)$ and $\left(X^{2}(1)=2.451, p=0.117\right)$, respectively.

No statistically significant differences were observed in patients' understanding of the radiologist's role after the intervention. Of 49 pre-intervention respondents, $77.6 \%$ reported that the radiologist "interpret[s] the images," $44.9 \%$ reported that the radiologist "run[s] the machine that takes the images," and $18.4 \%$ reported that the radiologist "maintain[s] the machine that takes the images." Of 36 follow-up respondents, $77.8 \%$ reported that the radiologist interpreted the images $\left(X^{2}(1)=0.001, p=0.980\right) ; 30.6 \%$ reported that the radiologist ran the machine $\left(X^{2}(1)=1.797, p=0.180\right)$; and $8.3 \%$ reported that the radiologist maintained the machines $\left(X^{2}(1)=1.723, p=0.189\right)$.

Approximately $1 / 3$ (31.3\%) of 48 respondents expected to meet the radiologist during the knee MRI, while $37.5 \%$ did not, and $31.3 \%$ were unsure. Twenty-two per cent $(22.2 \%)$ of the 36 follow-up respondents reported that they met the radiologist during their knee MRI, and $2.8 \%$ (one patient) were unsure. 


\section{Cureus}

\begin{tabular}{|c|c|c|c|}
\hline Radiologist Role or Characteristic & Pre-Intervention & Post-Intervention & P-value \\
\hline \multicolumn{4}{|c|}{ Radiologist run[s] the machine that takes the images. } \\
\hline Yes & $22(44.9)$ & $11(30.6)$ & \multirow{2}{*}{.180} \\
\hline No & $27(55.1)$ & $25(69.4)$ & \\
\hline \multicolumn{4}{|l|}{ Radiologist interpret[s] the images. } \\
\hline Yes & 38 (77.6) & $28(77.8)$ & \multirow{2}{*}{.980} \\
\hline No & 11 (22.4) & $8(22.2)$ & \\
\hline \multicolumn{4}{|c|}{ Radiologist maintain[s] the machine that takes the images. } \\
\hline Yes & $9(18.4)$ & $3(8.3)$ & \multirow{2}{*}{.189} \\
\hline No & $40(81.6)$ & $33(91.7)$ & \\
\hline \multicolumn{4}{|l|}{ Radiologist is a medical doctor. } \\
\hline Yes & $23(47.9)$ & $25(69.4)$ & \multirow{3}{*}{.120} \\
\hline No & $18(37.5)$ & $9(25.0)$ & \\
\hline I don’t know & 7 (14.6) & $2(5.6)$ & \\
\hline
\end{tabular}

TABLE 2: Pre- and post-intervention understanding of radiologists' role in patient care

\section{Imaging experience}

The vast majority (75.5\%) of the 49 respondents expected to have their imaging results within two days after the exam ( $22.4 \%$ same day, $24.5 \%$ one day after, $28.6 \%$ two days after). $10.2 \%$ expected results in one week; the few remaining respondents were spread between three and five days.

After the intervention, most (83.3\%) of the 36 follow-up respondents expected future outpatient medical imaging results by two days after the exam (11.1\% same day, $27.8 \%$ one day after, $44.4 \%$ two days after), $X^{2}(30)=75.663, p<0.001$ for all response options).

\section{Communication with the radiologist}

The vast majority (85.4\%) of 48 respondents wanted to be able to contact the person who read their exam, with a preference for email ( $51 \%$ of 49 respondents) and MyChart ${ }^{\circledR}$ ( $44.9 \%$ of 49 respondents). In-person and phone communication (32.7\% and $36.7 \%$, both of 49 respondents) were less preferred. One patient wanted to communicate with the person reading the imaging "through the ordering doctor." MyChart ${ }^{\circledR i}$ is the patientfacing component of the institution's electronic health record through which patients can communicate with physicians.

Only one patient called the radiologist and discussed results for 12 minutes. However, six of 36 follow-up respondents reported that they had contacted the person "who interpreted/read your exam:" two in person, one by email, three by phone, and one by "other" but did not specify (one person used two methods of contact).

\section{Nonresponse bias analysis}

The study's demographic evaluation found no evidence of nonresponse bias based on age (Mann-Whitney $\mathrm{U}$ test for non-normal data, $p=0.297)$ and gender $\left(X^{2}(1)=1.070, p=0.308\right)$.

\section{Validity and reliability analysis}

The study opted to evaluate validity with Cronbach's more traditional measures because of the binomial and cognitive nature of our questionnaires. Content validity was carried out through evaluation by a survey expert (AP) in addition to several attending radiologists who all had more than ten years of experience. The dichotomous data did not lend itself to a Cronbach's alpha for internal consistency analysis; however, several expected relationships existed that support the construct validity. Specifically, those who thought that a radiologist runs the machine that takes the images were more likely to expect to meet the radiologist $\left(X^{2}(2)=20.028, p<0.001\right)$ in the pre-appointment questionnaire and report that they did meet the radiologist 
during the exam $\left(X^{2}(2)=15.818, p<0.001\right)$ in the post-appointment questionnaire. Additionally, respondents who answered that a radiologist interpreted the images were more likely to also respond that the radiologist is a physician in both the pre $\left(X^{2}(2)=7.602, p=0.022\right)$ and post $\left(X^{2}(2)=17.589, p<0.001\right)$ appointment questionnaires.

\section{Discussion}

Our study found that many patients, despite being a highly educated cohort, did not understand radiologists' role in their care, a finding that did not improve significantly after a personal introductory communication from the radiologist. We also found discordance between patients' expressed desire to communicate with their radiologist and their actual rate of personal communication with their radiologist.

The first-round survey results re-demonstrated several current trends in the radiology literature. First, concordant with the recent trends toward patient autonomy and medical record access, patients desire access to their medical records--including radiology reports--and desire short turnaround intervals between completion of a radiologic examination and production of a report. Interestingly, although the vast majority of participants felt that it was desirable for the physician interpreting the examination to be available for discussion, only one out of 49 participants actually did engage with the radiologist. This suggests that perhaps the availability of the radiologist is reassuring in that it provides patients with an option, but ultimately patients are generally satisfied with the potential for the encounter with the radiologist without actually needing to engage in it. This is an area in which further exploration is needed.

Public awareness of the role of the radiologist is limited, with only a subset of patients correctly identifying the role of the radiologist in recent surveys addressing this issue $[7,10]$. In our pre-intervention survey, only $23 \%$ of respondents reported that the radiologist is a medical doctor. Interestingly, when the level of education was compared to responses to this question, an inverse correlation between education and knowing that radiologists are physicians was found. Simply put: the more educated a patient was, the less likely s/he was to know that a radiologist is a physician. This lack of understanding regarding the role of the radiologist may contribute to limited patient enthusiasm for consultation with the radiologists who read their imaging in our study. The knowledge gap is likely present because radiologists tend to operate outside of direct patient interaction. However, it is not clear from our intervention or survey why increasing levels of education are associated with the misunderstanding.

Although the vast majority of surveyed patients wanted to be able to contact the person who read their knee MRI, only one patient actually did reach out to the radiologist to discuss findings. Interestingly, six of 36 follow-up respondents reported that they had contacted the person "who interpreted/read your exam:" two in person, one by email, three by phone, and one by unspecified "other." It is likely that these patients communicated with the ordering physician, thinking s/he interpreted the image since that has historically been the patient's source for imaging results. The number also aligns relatively well with the approximately $25 \%$ of our patients who did not think the radiologist was the person interpreting the imaging. Alternatively, the patients are mistaking other radiology staff for the radiologist. Technicians should be reminded to properly introduce themselves and their role to ensure patients' understanding.

The fact that patients expressed a desire to communicate with the person reading their reports, but then did not take advantage of the opportunity to contact the radiologist, suggests that the issue is more complicated than just a lack of a pathway for communication between patients and radiologists. Perhaps the lack of clear understanding of the role of the radiologist hinders patients from contacting radiologists since they feel uncertain as to whom they are actually attempting to reach. Or perhaps patients are sufficiently reassured by just having a means through which they could contact the radiologist, and do not require the actual communication in order to feel comfortable.

\section{Limitations}

This study was limited by its single-institution design and relatively small sample size; the follow-up survey had a relatively low response rate as well. Additionally, patients were only screened for inclusion criteria three days a week, and many scans were conducted in less than 24 hours, excluding them from participation. It is also possible that there would be different results with a different imaging modality or body region.

\section{Conclusions}

Since a personalized communication to patients for a specific exam was unsuccessful in educating patients, ultimately informing patients about the vital role that radiologists contribute to their care and encouraging patients to make radiologists an active member of their healthcare team may be very difficult in the current healthcare model and should concern the radiology community. Future research should delve into why patients are not actually communicating with their radiologist when given the opportunity and explore working with colleagues in other specialities to help the radiology community inform patients of their care role. 


\section{Cureus}

\section{Appendices}

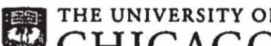 \\ CHICAGO \\ MEDICINE \& \\ BIOLOGICAL \\ SCIENCES
}

Hello,

It was a pleasure to have had the opportunity to be a part of your care here at the University of Chicago as a consulted physician. Thank you for participating in our University of Chicago Radiology study of patient engagement in radiology care and greater access to the radiologist that read your study. We take great pride in producing and interpreting the highest quality exams, often faced with challenging and less routine patient circumstances.

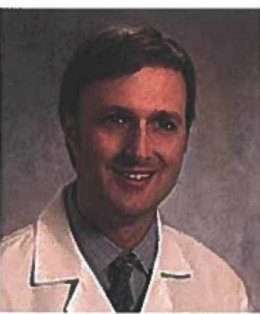

The attached report is your official diagnostic report which has already been posted electronically to your referring physician and your paperless electronic medical record (MyChart). We understand that not all of the vocabulary or reporting structure is readily understood, but these conventions are in place to maximize the efficiency and accuracy of your care between consulting physicians.

Please note that the full extent or implications of the MR study incorporates multiple factors, including a deeper understanding of your specific medical history, a physical exam, and possibly additional tests. This global understanding will be relayed to you by your primary ordering physician, along with treatment options which I may not be aware.

If you have specific questions about your knee MRI and experience, I can be only too pleased to assist you. I can be reached at 773-702-1607 during my office hours on Thursday, between 10:00AM and 12:00PM. I will do my best to address your immediate questions as they pertain to your MRI experience and your diagnostic report.

Thank you again for choosing the University of Chicago for your care and for completion of the pending short follow-up survey.

Sincerely,

Christopher M Straus M.D.

Associate Professor - Dept. of Radiology 


\section{Cureus}

PI: Christopher Straus, M.D.

Co-Investigators: Larry Dixon, M.D., Gregory S. Stacy, M.D., Stephen Thomas, M.D. Title: Optimizing the Radiology Experience through Radiologist-Patient Interaction

Appendix VI

Bucksbaum Research Project

Optimizing the Radiology Experience through Radiologist-Patient Interaction

retrieved:

\section{Patient Contact Information Form}

Date patient contacted you:

How long did you spend communicating with the patient? minutes

Why did the patient contact you and how much time did you spend on the topic? Check any of the boxes below

\begin{tabular}{|l|l|l|l|l|}
\cline { 2 - 5 } \multicolumn{1}{c|}{} & $\begin{array}{c}\text { Very little of the } \\
\text { call }\end{array}$ & Some of the call & Most of the call & All of the call \\
\hline Explain the findings & & & & \\
\hline $\begin{array}{l}\text { Explain the } \\
\text { vocabulary }\end{array}$ & & & & \\
\hline Course of treatment & & & & \\
\hline Other: & & & & \\
\hline Other: & & & & \\
\hline Other: & & & & \\
\hline
\end{tabular}

Additional comments:

Page $\mathbf{1 7}$ of $\mathbf{1 7}$

FIGURE 2: Patient contact information form 


\section{Cureus}

Confidential

Pre-appointment Survey

Page 1 of 3

This is the first of two surveys for a University of Chicago research study, Optimizing the Radiology Experience through Radiologist-Patient Interaction.

We thank you for your participation in this study and remind you that your participation is purely voluntary and if you choose to opt-out of the study, you may do so at any time and your care will not be impacted.

If you are still interested in participating in our study, please complete the following questions. The survey should take 4 to 6 minutes to complete.

Have you ever had any medical imaging before?

$O$ Yes
ONo

(Examples of medical imaging: MRI, x-rays, CT (cat) scans.)

Have you ever had an MRI before?

Yes
No

What is your highest level of education completed?

Less than a high school degree

High school degree or graduate equivalent degree (GED)

Some college*

Associates degree

Bachelor's degree

Graduate degree

$\left({ }^{*}\right.$ please select 'Some college' if you are

currently an undergraduate student)

When do you expect to have the results of your upcoming MRI of the knee?

Same day

the day after the exam

2 days after the exam

3 days after the exam

4 days after the exam

5 days after the exam

6 days after the exam

$\bigcirc 1$ week after the exam

FIGURE 3: Pre-appointment survey 1 of 3 


\section{Cureus}

Confidential

Page 2 of 3

\begin{tabular}{|c|c|}
\hline What does a radiologist do? & $\begin{array}{l}\square \text { Run the machine that takes the images } \\
\square \text { Interpret the images } \\
\square \text { Maintain the machine that takes the images } \\
\square \text { Other } \\
\text { (Please select all that apply.) }\end{array}$ \\
\hline If other, what do you believe a radiologist does? & \\
\hline Will you meet the radiologist during your knee MRI? & $\begin{array}{l}\text { Yes } \\
\text { O No } \\
\text { O I don't know }\end{array}$ \\
\hline Is a radiologist a medical doctor? & $\begin{array}{l}\text { Y Yes } \\
\text { ONo } \\
\text { OII don't know }\end{array}$ \\
\hline
\end{tabular}

FIGURE 4: Pre-appointment survey 2 of 3 


\section{Cureus}

Confidential

Page 3 of 3

\begin{tabular}{|c|c|}
\hline Do you know how to access your MRI exam result? & $\begin{array}{l}\text { OYes } \\
\text { O No }\end{array}$ \\
\hline $\begin{array}{l}\text { Would you like to be able to contact the person who } \\
\text { read your exam? }\end{array}$ & $\begin{array}{l}\text { OYes } \\
\text { No }\end{array}$ \\
\hline $\begin{array}{l}\text { If yes, how would you prefer to communicate with him } \\
\text { or her? }\end{array}$ & $\begin{array}{l}\square \text { In person } \\
\square \text { Telephone } \\
\square \text { Email } \\
\square \text { MyChart } \\
\square \text { Other } \\
\text { (Please select all that apply.) }\end{array}$ \\
\hline
\end{tabular}
him or her?

FIGURE 5: Pre-appointment survey 3 of 3 


\section{Cureus}

Confidential

Post-appointment Survey

Page 1 of 2

This is the second and final survey for a University of Chicago research study, Optimizing the Radiology Experience through Radiologist-Patient Interaction.

We thank you for your participation in this study and remind you that your participation is purely voluntary and if you choose to opt-out of the study, you may do so at any time and your care will not be impacted.

If you are still interested in participating in our study, please complete the following questions. The survey should take 4 to 6 minutes to complete.

For future outpatient medical imaging, when do you expect to have the results of your exam?

Same day

the day after the exam

02 days after the exam

3 days after the exam

4 days after the exam

5 days after the exam

6 days after the exam

What does a radiologist do?

Run the machine that takes the images Interpret the images

Maintain the machine that takes the images

Other

If other, what do you believe a radiologist does?

Did you meet the radiologist during your knee MRI?

YYes

No

O I don't know

Is a radiologist a medical doctor?

Y Yes

OIdon't know

Do you know how to access your MRI exam result?

Yes
No

FIGURE 6: Post-appointment survey 1 of 2 


\section{Cureus}

\author{
Did you contact the person who interpreted/read your \\ exam? \\ If yes, how did you communicate with him or her?
}

If other, how did you contact him or her?

If no, how would you have liked to communicate with him or her?

If other, how else would you prefer to contact him or

her?
Y Yes
No

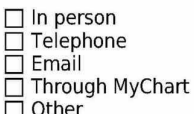

Through

(Please check all that apply.)

In person

In person
Telephone

Email

Through MyChart

(Please select all that apply)

FIGURE 7: Post-appointment survey 2 of 2

\section{Additional Information \\ Disclosures}

Human subjects: Consent was obtained by all participants in this study. University of Chicago IRB issued approval n/a. exemption status. Animal subjects: All authors have confirmed that this study did not involve animal subjects or tissue. Conflicts of interest: In compliance with the ICMJE uniform disclosure form, all authors declare the following: Payment/services info: All authors have declared that no financial support was received from any organization for the submitted work. Financial relationships: All authors have declared that they have no financial relationships at present or within the previous three years with any organizations that might have an interest in the submitted work. Other relationships: All authors have declared that there are no other relationships or activities that could appear to have influenced the submitted work.

\section{References}

1. Johnson AJ, Easterling D, Williams LS, Glover S, Frankel RM: Insight from patients for radiologists: improving our reporting systems. J Am Coll Radiol. 2009, 6:786-794. 10.1016/j.jacr.2009.07.010 
2. Johnson AJ, Frankel RM, Williams LS, Glover S, Easterling D: Patient access to radiology reports: what do physicians think?. J Am Coll Radiol. 2010, 7:281-289. 10.1016/j.jacr.2009.10.011

3. Johnson AJ, Easterling D, Nelson R, Chen MY, Frankel RM: Access to radiologic reports via a patient portal: clinical simulations to investigate patient preferences. J Am Coll Radiol. 2012, 9:256-263.

10.1016/j.jacr.2011.12.023

4. Safdar N, Shet N, Bulas D, Knight N: Handoffs between radiologists and patients: threat or opportunity? . J Am Coll Radiol. 2011, 8:853-857. 10.1016/j.jacr.2011.08.010

5. Basu PA, Ruiz-Wibbelsmann JA, Spielman SB, Van Dalsem VF 3rd, Rosenberg JK, Glazer GM: Creating a patient-centered imaging service: determining what patients want. AJR Am J Roentgenol. 2011, 196:605610. 10.2214/ajr.10.5333

6. Mangano MD, Rahman A, Choy G, Sahani DV, Boland GW, Gunn AJ: Radiologists' role in the communication of imaging examination results to patients: perceptions and preferences of patients. AJR Am J Roentgenol. 2014, 203:1034-1039. 10.2214/ajr.14.12470

7. Koney N, Roudenko A, Ro M, Bahl S, Kagen A: Patients want to meet with imaging experts . J Am Coll Radiol. 2016, 13:465-470. 10.1016/j.jacr.2015.11.011

8. Cabarrus M, Naeger DM, Rybkin A, Qayyum A: Patients prefer results from the ordering provider and access to their radiology reports. J Am Coll Radiol. 2015, 12:556-562. 10.1016/j.jacr.2014.12.009

9. Henshaw D, Okawa G, Ching K, Garrido T, Qian H, Tsai J: Access to radiology reports via an online patient portal: experiences of referring physicians and patients. J Am Coll Radiol. 2015, 12:582-586. 10.1016/j.jacr.2015.01.015

10. Kuhlman M, Meyer M, Krupinski EA: Direct reporting of results to patients: the future of radiology? . Acad Radiol. 2012, 19:646-650. 10.1016/j.acra.2012.02.020

11. Halbesleben JR, Whitman MV: Evaluating survey quality in health services research: a decision framework for assessing nonresponse bias. Health Serv Res. 2013, 48:913-930. 10.1111/1475-6773.12002 